Results Although inequalities in a healthy lifestyle were found among men and women for both indicators, variations in their direction and magnitude for incident disease risks occurred. Diabetes incidence was inversely associated with both indicators in multivariate models, but adjustment for Body mass index substantially attenuated the associations. Middle-educated women had a lower risk for myocardial infarction (HR 0.39, 018-0.84), but a higher risk for stroke (HR 2.03, 1.07-3.83), in fully-adjusted models. Protective effects against cancer were shown for semiprofessional men and unskilled women, but not for education. Also the results of the literature search showed that different socioeconomic indicators appeared to vary in their associations to chronic disease risk.

Conclusions Educational level and occupational social class varied in their influence on incident chronic diseases, but are less important predictors for disease risk in the EPIC-Potsdam study.

\section{P2-517 SOCIOECONOMIC STATUS (SES) RELATED TO BONE PROPERTIES IN CANADIAN ADOLESCENT FEMALES?}

doi:10.1136/jech.2011.142976m.44

S Imam, M Tammemagi, ${ }^{*}$ P Klentrou, K Gammage. Brock University, St. Catharines, Ontario, Canada

Introduction Osteoporosis is a bone condition characterised by low bone mass and increased susceptibility to fractures in older adults, particularly females. Peak bone mass (PBM) is accrued by age twenty in females. It is imperative adequate PBM be acquired in adolescent females to minimise future risk for osteoporosis. There is limited literature on the relationship between SES as a risk factor and bone properties in adolescent females.

Methods In a cross-sectional study of Canadian adolescent females $(\mathrm{n}=412)$ from six randomly selected schools in Southern Ontario, multivariable regression analyses were used to assess the association between aggregate SES indices and bone speed of sound (SOS) in a multilevel model, participants nested in schools. Bone SOS was measured by transaxial quantitative ultrasound at the distal radius and mid-tibia. SES was determined by matching residential address for each participant with Statistics Canada 2006 Census data for their dissemination area.

Results Mean age was $15.7 \pm 1.1$ years. Multilevel analysis found a significant difference in SOS among schools at both radial and tibial sites $(p<0.001)$. Multivariable regression analysis indicated significant positive relationships for median family income $(p=0.036)$ and median household income $(p=0.017)$ with tibial SOS adjusted for grade, weight, body mass index, smoking and alcohol use. Further analysis of average family income $(p=0.035)$ and average household income $(p=0.017)$ also indicated significant positive relationships with tibial SOS.

Conclusion These data suggest school and SES at census aggregate variable level are important predictors for bone SOS in female adolescents, school appearing to dominate SES variables.

\section{P2-518 SORGHUM CONSUMPTION MODIFIES THE EFFECT OF FLUORIDE ON DENTAL FLUOROSIS IN INDIA}

\section{doi:10.1136/jech.2011.142976m.45}

${ }^{1} \mathrm{C}$ Janakiram, ${ }^{*}{ }^{2} \mathrm{~K}$ R Thankappan, ${ }^{3} \mathrm{~K}$ R Sundaram. ${ }^{1}$ Amrita School of Dentistry, Cochin, Kerala, India; ${ }^{2}$ Achutha Menon Centre for Health Science Studies, Sree Chitra Tirunal Institute for Medical Sciences and Technology, Trivandrum, Kerala, India; ${ }^{3}$ Amrita School of Medicine, Cochin, Kerala, India

Background Dental fluorosis is a major public health problem in 17 states of India. Earlier studies reported that sorghum \{a type of millet, (jowar) $\}$ consumption interacts with fluoride in the body and enhances fluorosis.
Objectives This study was carried out to explore the potential association between sorghum consumption and severity of dental fluorosis.

Methods A community based case control study was carried out in villages having different fluoride levels (high, medium and low) in drinking water in Davangere, India. 352 school Children (12-15 years, male $58 \%$ ) with severe grades of dental fluorosis classified by Thylstrup \& Fejerskov Index (scores 4-9) were selected as cases. 428 school children (12-15 years, male 48.8\%) with no dental fluorosis were selected randomly from the same area as controls. Exposure ascertainment of jowar consumption was done by 24-h diet recall and food frequency questionnaire. Logistic regression analysis was done using SPSS version 17.

Results Children who consumed jowar had 2.67 times more chance of getting severe dental fluorosis compared to those who did not (OR 2.67, CI 1.98 to 3.62). The ORs for jowar consumption and dental fluorosis were 1.59, 3.18 and 3.76 at each stratum, for low, medium and high fluoride villages respectively. The MantelHaenszel OR was 2.58 ( $p$ value $=0.001$ ) which was similar to crude OR. The test of homogeneity (Breslow test) showed $\mathrm{p}$ value $=0.05$. Conclusions Sorghum consumption modifies the effect of fluoride in occurrence of severity of dental fluorosis in this population.

\section{P2-519 EPIDEMIOLOGY OF INJURY IN RURAL PONDICHERRY, INDIA}

doi:10.1136/jech.2011.142976m.46

G Kalaiselvan,* A Dongre, T Mahalakshmy. Department of Community Medicine, Sri Manakula Vinayagar Medical College, Pondicherry, India

Objective To find out the prevalence of "all" injuries, its nature, outcome and sources of treatment among rural population of Pondicherry.

Methods It was a triangulated study of quantitative (survey) and qualitative (Focus Group Discussion, FGD) methods. The trained second year medical undergraduate students paid house visits to all houses in five feasibly selected villages of our field practice area. The students interviewed the housewife and obtained information for all injuries for each family member in last one year and its sources of treatment. We could obtain information for 1613 (96.7\%) households. Post-survey, FGDs were undertaken to explore the various traditional treatments for the common injuries. The data were entered and analysed using Epi_info 6.04d software package.

Results Overall, the prevalence of all injury among all age groups was $30.6 \%$ in last one year. Injuries were significantly more after 18 years of age and among men $(p<0.001)$. About $99.2 \%$ injuries reported were accidental and majority (58.2\%) went to government doctor for treatment. Most common causes of injuries were fall on the ground from height or due to slip (7.4\%), road traffic accidents $(5.6 \%)$, agriculture related injuries $(5 \%)$ and bites by scorpion/ insects/snakes/dogs (4.1\%). FGDs explored some potentially harmful traditional remedial measures at village level.

Conclusions Considering the high prevalence of all injuries related to road traffic accidents, fall from height and agriculture work related injuries across all age groups, especially among men and some potentially harmful traditional treatments, an intervention in the form of targeted injury prevention program is required.

\section{P2-520 INJURY- RELATED MORTALITY AMONG WOMEN AGED 12-49 YEARS: DEMOGRAPHIC AND HEALTH SURVEY (DHS) PAKISTAN}

doi:10.1136/jech.2011.142976m.47

U Khan, ${ }^{*}$ A Hashmi, N Zia, S Awan, J Razzak. Aga Khan University, Karachi, Pakistan

Introduction Beyond maternal health, there are other issues of women health like injury prevention that adequately need to be 